\title{
The Power of Consumer Activism and the Value of Public Health Immunization Registries in a Pandemic: Preparedness for Emerging Diseases and Today's Outbreaks
}

\author{
Michael L. Popovich, MSSE ${ }^{*}$, Todd Watkins ${ }^{1}$, BSIE $^{1}$, Ousswa Kudia, MPH${ }^{1}$
}

1. Scientific Technologies Corporation, 411 S. $1^{\text {st }}$ Phoenix, AZ 85004.

\begin{abstract}
Public Health immunization registries and the immunization ecosystem have evolved over the past two decades to become significant population health data assets. Clinical providers and pharmacists are reporting the immunizations given to their patients to public health registries in 49 states and all territories, creating consolidated immunization event patient records.

Most of these immunization events are reported through the provider's Electronic Health Record system (EHR), Pharmacy Management System (PMS), online, or through data uploads. Meaningful Use and health data standards (HL7) became the drivers that accelerated reporting to immunization registries and significantly improved the quantity and quality of the data. The infrastructure supporting the Immunization Ecosystem (IE) has enabled real-time compliance reporting and, more importantly, realtime patient queries. The provider community now has online access to a patient's immunization history in over three quarters of the states, and growing. This access includes a forecast of the patient's immunization gaps provided by public health decision support tools based upon the most recent ACIP recommendations. This is creating an opportunity for the provider and the patient to work together to reduce their risk of suffering a vaccine-preventable disease. This IE and the data in an Immunization Information System (IIS) are especially useful as pharmacies expand their immunization practices and create opportunities to reduce the adolescent and adult immunization gaps.

In a few states, this provider-public health ecosystem has begun to extend to individuals by allowing them to access the IIS online through the use of MyIR. MyIR provides them with the electronic version of their immunization "yellow cards," recommendations for immunizations due, and the ability to print official certificates. This emerging consumer engagement creates opportunities to empower individuals to be more proactive in their family's health care.

This paper builds upon early experiments to empower individuals in this ecosystem by leveraging the value of these public health data assets and trusted communications, illustrating the possibilities for engaging consumers to support reducing the impact of emerging diseases, outbreaks and the next pandemic. This paper will suggest the value of the IE and the role individuals can play within their own social networks to advance public health efforts to manage disease events. In turn, this social mission would encourage consumers to be more proactive in managing their own healthcare.

Keywords: Immunization Information System, Pandemic, Disease X, Consumer Engagement, Consumer Empowerment, Public Health Registries, Preparedness, Immunization Ecosystem, Health Information Technologies, Vaccinations, Vaccines
\end{abstract}

Online Journal of Public Health Informatics * ISSN 1947-2579* http://ojphi.org * 10(2):e203, 2018 
Abbreviations: World Health Organization (WHO), Crimean-Congo Haemorrhagic Fever (CCHF), Middle East Respiratory Syndrome Coronavirus (MERS-CoV), Rift Valley Fever (RVF), Health Information Technology (HIT), Electronic Health Record (EHR), Health Level 7 (HL7), Severe Acute Respiratory Syndrome (SARS), Scientific Technologies Corporation (STC), My Immunization Record (MyIR), Immunization Information System (IIS), Centers for Disease Control and Prevention (CDC), Centers for Medicare \& Medicaid Services (CMS), Meaningful Use (MU), Office of the National Coordinator for Health Information Technology (ONC), Pharmacy Management System (PMS), Immunization Ecosystem (IE), Advisory Committee on Immunization Practices (ACIP).

*Correspondence: michael popovich@stchome.com

DOI: 10.5210/ojphi.v10i2.9147

Copyright (C2018 the author(s)

This is an Open Access article. Authors own copyright of their articles appearing in the Online Journal of Public Health Informatics. Readers may copy articles without permission of the copyright owner(s), as long as the author and OJPHI are acknowledged in the copy and the copy is used for educational, not-for-profit purposes.

\section{Introduction}

When asked what scared him the most and kept him up at night, Tom Frieden, the former Director of the CDC, replied: "The biggest concern is always for an influenza pandemic." [1]

A recent CNN article [2] outlined the World Health Organization's (WHO) review of potential public health emergency diseases that included the top 10 global concerns: Crimean-Congo haemorrhagic fever (CCHF), Ebola virus disease and Marburg virus disease, Lassa fever, Middle East respiratory syndrome coronavirus (MERS-CoV) and Severe Acute Respiratory Syndrome (SARS), Nipah and henipaviral diseases, Rift Valley fever (RVF), Zika, and finally Disease X. Currently there are no vaccines to prevent these. The WHO's Disease $\mathrm{X}$ was included in this "List of Blueprint priority diseases" [3] because the world does not know what pathogen can cause the next epidemic.

Globally, as well as in the U.S., epidemiologists and public health professionals work endlessly to ensure the risk and impact of existing and emerging diseases are minimized, and that neither turns into a pandemic. As the world increasingly becomes interconnected through travel and technology, timely information and accurate data become more imperative. Early warning of disease occurrence and assessing the resulting impact on the public are paramount. Early warning systems referred to as "syndromic surveillance," along with mandated notifiable disease reporting, capture data and electronically process the information to present public health with a view into future potential impacts.

Over the past two decades, new information systems have played a key role in improving public health's early warning and case management for disease outbreaks. As improved analytics are used to predict risk in populations, researchers and epidemiologists open new doors to disease cures, clinical research develops new medicines, and providers develop new care models. The role of technology and public health to support these efforts therefore becomes more valuable.

Health information systems are traditionally considered to be used for electronic medical record or payer billing systems. They are not paired with technology advancements that exist in the 
hands of consumers which could encourage patients to be more proactive with their healthcare. Efforts today to link information and technology to engage consumers are championed by health plans and healthcare providers. Engaging and empowering individuals to be proactive when presented with their medical records is not a simple problem to solve. It is not just a matter of making data available but making it actionable. Actionable information may not achieve the desired success.

But what if... this was augmented with another social mission? What if... the health community engaged consumers to help them fight disease outbreaks; what if... consumers become frontline activists to report occurrences and outcomes, and become "intelligent connections" to extend the right information in their social networks? This would encourage today's consumer technology to be better integrated with the clinical health information technology (HIT). It would encourage continued investment in evolving and sustaining critical public health ecosystems. It would create opportunity to engage consumers, empowering each to be more proactive in supporting population health and their own healthcare.

Another large part of the health information system are immunization information systems (IIS) where individuals who have received vaccines are documented in a confidential computer based system in a specific geographic area [4]. The IIS can be used for disease surveillance purposes and provide valuable information to public health authorities [5]. As an extension of one of the existing IISs, MyIR was created where any state IIS, pharmacy or provider can provide patients direct access to family state immunization records - regardless of the type of immunization information system used. Providers can communicate to patients using MyIR to increase patient engagement. Automated vaccine reminders can be sent using this system as well.

\section{Purpose}

During a pandemic, the need for accurate and timely information is vital. We propose that if there were direct public health agency communication channels to individuals - by building on existing immunization networks, the public would receive correct information quicker. Furthermore, there is value that can be leveraged from social networks to advance public health efforts to manage disease events and in turn encourage consumers being more proactive in managing their own health care.

\section{Method}

One could envision a public-health-engagement-approach to empower consumers begins by offering individuals a challenge and a mission they care about. A mission that allows them to contribute to the social good with the added benefit of making them more attentive to their own healthcare. Placing this mission in the palms of their hands through every cell phone is the first objective. The second is to create value for this phone's owner to become active in supporting the mission. The third objective is to provide a commonly understood health event that is a cornerstone component to this phone owner's health and welfare that helps launch them toward utilizing their complete health information profiles.

Universally the most significant public health event in the 20th century was the power of vaccines and applied immunizations to individuals and large populations [6]. 
The most significant action an individual can take to reduce their risk of a vaccine-preventable disease is to become vaccinated and stay up-to-date on their immunizations.

However, there is a significant gap between believing in the value of an immunization and in ensuring one's own (and one's family's) immunizations are current. How many individuals really understand what the immunization schedule recommends or are proactive in ensuring they have no immunization gaps? How many of these same individuals in an outbreak or with news of a new disease ask the question "Is there a vaccine and what are my risks?"

The challenge is how to engage individuals directly, empower them to be advocates for their own health and in an outbreak become sources of trusted public health messages as they communicate within their social network, effectively supporting the higher-level mission. The lesson learned from every outbreak? That the public demands accurate, timely, and transparent communication from the government. If this public health information is communicated directly to this new evangelistic network, there is the potential to expand to larger consumer networks.

A few separate experiments were conducted using MyIR.

\section{Increase Sustainment}

The first experiment aimed to increase sustainment. The engagement project was to reach out in an effort to increase sustainment by contacting users who had not used MyIR and accessed their data from the IIS in over 30 days. The baseline looked at number of users that were logging into MyIR more than once a month. The target was non-engaged users. In this category there were nearly 8,000 accounts.

\section{Flu Shot Customer Engagement}

The second experiment aimed to engage the consumer for the flu shot.

For this campaign, on November 21st, 2017, an email was sent to 7,772 users that asked them a simple question, "Did you get your flu shot?" If they selected Yes, they saw a funny meme and received positive affirmation. If they clicked No, the message was an encouragement to get their flu shot before Thanksgiving 2017.

\section{Customer Engagement-Healthy Lifestyle}

In the third experiment, consumer engagement effort was initiated January 19, 2017. Capitalizing on the popularity of New Year's resolutions around wellness this engagement experiment created a Healthy Lifestyle page within MyIR. It featured an Arizona food blogger, Simple, Sassy and Scrumptious, who offers readers nutritious easy meal ideas.

\section{Outreach Efforts}

The fourth experiment focused on outreach efforts where Louisiana targeted users who had failed to complete the two step process to fully enroll for access to their immunization histories. 


\section{Efficacy of Flu}

The last experiment was in April 2018, where a MyIR outreach initiative in Washington and Louisiana asked two questions to a sample of 212 consumers who access their records from the state IIS: Did you get a flu shot this year? Do you feel like you got the flu this year?

\section{Trusted Digital Communication}

Consider the limitation of traditional media coverage of disease events; whether it is the efficacy of this year's flu vaccine, reporting on the occurrence of a previously eliminated vaccine preventable disease or coverage of an emerging disease from Zika to Disease X. Common communication through media and healthcare-related web sources rely on consumer education campaigns that include uncontrolled dissemination and information exchange after the initial message. A more optimal approach is to extend this message from trusted public health sources to individuals.

Trusted communication links exist. An example is retail pharmacies. These trusted digital communication channels are used every day by pharmacists to notify their patients of prescriptions, immunizations due, and results of point-of-care testing. If these channels were leveraged to support major public health events, a national scalable digital trusted social network would extend across the country to achieve the desired result. Building this communication network on an existing national framework would leverage prior investments and day-to-day operational processes. The question is, does such a network currently exist? The answer is Yes and it is one that is growing every day. One that was created by the need to reduce the impact of disease. It is the national immunization ecosystem.

Forty-nine states have established immunization information systems (IIS) to capture and consolidate patient immunization events as reported by their clinicians or pharmacists [7]. HL7 is a set of standards for the transfer of clinical and administrative data between applications among the healthcare system's stakeholders. In nearly all these states, and soon all, secure HL7 data exchanges exist, allowing electronic health records (EHRs) and pharmacy systems to report a patient immunization event to the state IIS in real time. Furthermore, this infrastructure allows a clinician or pharmacist to review a patient's vaccine history and actionable Immunization Intelligence provided by public health decision support tools that using the latest Advisory Committee on Immunization Practices (ACIP) recommendations to identify immunization gaps and establish opportunities for closing these through communication at the patient point-of-care.

The immunization ecosystem is based on what public health has spent the past quarter century developing. This is based on statewide population health environments consisting of key data assets and technical infrastructure for reporting, accessing and assessing immunization activity. Additionally, these systems layer decision support, analytics, and communications across the entire population health environment. The value that the immunization ecosystem creates is the ability for all key "players" to integrate or connect. Thus, a national immunization ecosystem exists today and, as it continues to evolve, the framework and platform are primed to impact the cost of health care and improve patient outcomes. The key is to fully extend the framework to the consumer, creating end-to-end communication channels between trusted health authorities and individuals. 
Pandemic preparedness planning that takes advantage of IISs, their infrastructure and their players while engaging individuals to be consumer activists in an emergency response network, is potentially a new approach to accelerating individual health accountability while offering public health the ability to communicate valuable outbreak or pandemic information. By doing so, the outcome should influence day-to-day consumer behavior to mitigate the outbreak's impact on the population.

\section{Delivering the Right Message}

The 2003 SARS outbreak (with a 40-billion-dollar economic impact) [8], the 2009 H1N1 pandemic, and the 2015 Zika outbreak confirmed that the questions asked by the public in each of these events were:

- Is there a vaccine for this [9]?

- Am I or is my family at risk [10] [11]?

- Should I travel [10] [11]?

The ability for continued government and health authority responses to these questions is an important step toward managing the welfare of concerned populations while outbreaks are studied, and public health mitigation plans are put into place.

The velocity of information, both accurate and inaccurate, cannot be controllable through current communication channels available to disseminate information to individuals, especially those most at risk. Public health has an expectation and relies on their disease specific health education reaches at risk individuals, and is clearly understood and accepted. This reliability can be impacted by the social media and online consumer world today. Much of the public's information and behavior during a pandemic will be influenced through these social media and online networks [12]. Individuals will search for data they choose to believe [13]. Once found, they will push this information to their family and friends and their social media sphere of influence [14]. Information from social media can pose as a challenge to stability for the trusted information that public health wishes to deliver in order to inform and encourage those at risk to take action [15]. When a pandemic occurs, the need for accurate and timely information accelerates. If the odds of receiving accurate information during a pandemic are against you in the social media world, consider the opportunity if there were direct public health agency communication channels to individuals - by building on existing immunization networks. Accurate communications would be the goal during a public health event.

In Washington and Louisiana currently, over 35,000 consumers access their immunization records through real-time connections to the state IIS through MyIR. The "players" in these states encourage individuals to enroll for access. Once enrolled, an individual is connected to one of their health data attributes through the immunization platforms, which also establishes information access to trusted health agencies. It creates the opportunity to accelerate consumer engagement and activism in a pandemic or outbreak by layering digital communication to individuals to include those most at risk, and supporting the extension of this trusted communication through the individual's social network. 


\section{Communication Attributes to Empower}

Based on the experiences gathered in working with immunization data and health pandemics, the following steps are proposed to increase communication to the public during a health outbreak:

1. Using existing infrastructure for immunization data exchange, establish communication links to trusted public health authorities supporting the following actions:

a. Inform and engage consumers by proactively alerting and notifying each individual of their immunization coverage gaps.

b. Identify the nearest locations capable of providing specific immunizations and antivirals to high risk populations.

c. Capture feedback and monitor outcomes or concerns of individual immunization events at their time of occurrence as well as over an extended period of time.

d. Capture surveillance of personal (family) health events, such as influenza or influenza-like illness.

2. Integrate provider, pharmacy and laboratory patient influenza tests, public health influenza reporting, and overall tracking of the most recent outbreak.

3. Integrate outbreak occurrences through alerts and visual displays (risk and outbreak maps).

4. Provide social media exchanges to link Immunization Ambassadors and general social networks that can support the concept of "layering" to reduce the impact of an outbreak and to ensure a controlled, consistent and accurate dissemination of information.

\section{Illustrations: How the Immunization Ecosystem Supports Consumer Engagement}

Since 2016, a number of immunization consumer engagement campaigns have been undertaken by STC to explore the power of direct links to individuals who have enrolled and have access to their immunization records. In a 90-day period from mid-August 2016 to mid-November, the STC consumer tool MyIR had 15,000 total user visits to the state immunization registry. Of these 23\% were returning users. In that same period 3,200 users visited MyIR more than once a month. They spent an average of 5 minutes on the site each time and viewed seven different pages. Typically, this included the initial login, requests for their immunization records, and reviewing the forecast or immunizations due or past due for each family member.

In reviewing the individuals that enroll for user access to the immunization registry, they currently are from a specific demographic. The majority are within the ages of 25-44, with $72 \%$ being female. This suggests the people who see the most value in MyIR are likely to be women with a family. 


\section{Results}

\section{Increase Sustainment}

$27 \%$ of people opened the initial email and 3.6\% of these individuals used MyIR within 30 days to access their immunization records.

\section{Flu Shot Customer Engagement}

$9 \%$ answered the question with $80 \%$ of these saying "YES" they got their flu shot. As a result, 25 new immunizations were administered to these individuals within 60 days. One nine-month-old family member received a full series of age appropriate immunizations.

\section{Customer Engagement-Healthy Lifestyle}

A 7.1\% increase in returning users were tracked and a 9.1\% increase in engaged users, again defined as logging in more than once a month. There was also a $6.7 \%$ increase in average session duration.

\section{Outreach Effort}

Five hundred fifty-six emails were sent and a 30\% open rate was achieved. The email contained a single step to finalize the enrollment and thus access to their immunization medical records. Fifty of those who opened the email followed through and completed the process.

\section{Efficacy of Flu}

Respondents were asked: "Did you get a flu shot this year? Do you feel like you got the flu this year?" 78\% responded they did receive the flu shot this year. Of these $61.5 \%$ felt they got the flu this year which equates in this group to a $38.5 \%$ efficacy rate for this year's influenza vaccine. In February, CDC had determined the interim estimates for the effectiveness of the influenza were $36 \%[16]$.

These consumer engagements were early experiments. They initially targeted to increase consumer access and utilization of the information contained in a public health immunization registry. They have moved toward soliciting input from active users to test the concept of empowering advocates to support a larger public health mission and engage with trusted communications from government. They created the thought processes that lead to the concept of extending the IE to proactively engage individuals to support public health missions, notably outbreaks and the next pandemic. The data, technical frameworks and infrastructure of the IISs form the environment to engage and empower consumers to be field assets to support outbreak mitigation efforts and be instrumental within the social networks if a pandemic were to occur.

\section{Discussion}

Our aim was to illustrate examples where public health agencies using direct communication channels to individuals - by building on existing immunization networks, could increase the efficacy of reaching the public with correct information. The illustrations used to demonstrate the 
consumer engagement potential were not designed to test the hypothesis that consumer activism and the value of public health immunization registries in a pandemic would prove effective. They do demonstrate the potential of engaging individuals that have enrolled to access their immunization records from public health registries. They demonstrate that a subset of these individuals will provide information requested from public health authorities. It was through these early experiments and the growing data assets in state immunization systems that create a framework and technical platform to accelerate the potential value of engaging individuals in response plans for pandemic preparedness planning and support of today's outbreak.

The next step is to begin to engage individuals to establish those that would be willing to provide ongoing information to public health specific to immunizations and disease occurrences. This would include developing experiments that test a social campaign to engage these individuals over the course of a year to ensure they remain activists in this network and then initiate efforts to encourage them to be proactive in their health care. The impact would be monitored in order to begin to establish a model with key parameters that would allow scaling across states and different demographics of users.

The experiments illustrated were not statistically tested or compared to other sources. They were not designed to collect specific and more detailed information in support of an outbreak to determine if it is possible to enroll advocates and ensure consumer activism. However, sufficient assets and a growing community of individuals with access to their online immunization histories suggest a specific demonstration project to test this hypothesis is warranted.

\section{Limitations}

The paper presents a concept of empowerment and a few example consumer engagement tests that indicate the possible opportunity for public health. The results of the consumer engagements were not measured against a specific research goal to determine their effectiveness. There were no comparisons to other public outreach and education methods that would established the potential. The consumer outreach efforts were not regional, culturally, or demographic specific and as such there was no intent to determine the true public health education to populations approach. These are all recommendations for next steps although there is some justification to simply begin to enhance and expand the current process and develop a stronger strategy to be tested.

\section{Conclusion}

This is the start. The immunization ecosystem is a robust environment to build upon. Consolidated immunization information systems and technology supported by public-private partnerships have reached the dynamic where data and information is available across wide networks of users, stakeholders, and consumers. While health plans, providers and pharmacists struggle to engage their networks, by encouraging patients to be proactive in their healthcare, public health immunization assets may be the tipping point to accelerate this movement since the single most common health event is an immunization, required from birth to death.

Furthermore, pandemic preparedness planning is primed to step beyond just response actions and traditional communication plans to reach directly into areas where outbreaks are occurring, 
soliciting consumer activism through this ecosystem to report disease occurrences and disseminate accurate information as public health works to mitigate the outbreak. The hypothesis can extend one step further: If you are active in supporting a higher cause and technology is the facilitator, the belief is this would not only reduce the risk of outbreaks and help mitigate the economic impact and costs, but also create momentum for more people to become proactive with all their healthcare.

Continued investment through government immunization programs, Centers of Medicare and Medicaid Services (CMS) 90/10 match programs, Office of National Coordinator (ONC) efforts supporting innovation, and consumer empowerment are essential to continue to evolve and sustain the Immunization Ecosystem and data assets. As these assets create added value to each stakeholder, the government investment begins to create a positive return. The private sector benefits also grow. Their investment in this same ecosystem is necessary.

Today, opportunities to use this ecosystem to drive down healthcare costs and improve patient outcomes are unlimited. The value of this virtual ecosystem to the nation is untapped. Being ready for the next pandemic through everyday practice is unique. We are in unique times.

${ }^{1}$ New Hampshire is the only state that has not implemented an immunization registry, although they have one in test and expect to begin to implement this system in 2018.

${ }^{2}$ Players in this sense represent all key stakeholders that utilize immunization information and include public health, providers, pharmacists, payers, employers, school nurses, foster care, consumers, and more.

${ }^{3}$ For example, feedback such as: Did you receive this year's influenza immunization? Do you feel like you have the flu? (The dollar cost of the flu on the U.S. economy, according to CDC Health Affairs 2016-17, was $\$ 5.8$ billion.) In a Pandemic with a new vaccine, the question might be: Do you feel you had a reaction to the vaccine?

${ }^{4}$ Federal funding provided by ONC has partially supported the expansion and testing of STC's MyIR since 2014.

${ }^{5}$ Sustainment of public health immunization registries and technical infrastructure is the subject of a separate STC paper, "Sustaining the Public Health Immunization Ecosystem through Public Private Partnerships."

${ }^{6}$ The content and concepts of this paper are based upon STC's 25 years of working in the immunization registry sector, which includes implementing state public health IIS, implementing electronic HL7 connections to every state IIS from over 30,000 pharmacies and 10,000 providers, and using data and analytics to tell the story of the power of the ecosystem.

\section{Acknowledgements}

We would like to thank Theresa Munanga for her editorial assistance. We would also like to thank all STC employees, especially those who assisted with the MyIR studies. 


\section{References}

1. Sun LH, Outgoing CDC. Chief talks about agency's successes-and his greatest fear. The Washington Post [Internet]. 2017 Nov [Accessed 2018 Jun 01]. Available from: https://www.washingtonpost.com/news/to-your-health/wp/2017/01/16/outgoing-cdc-chieftalks-about-the-agencys-successes-and-his-greatestfear/?noredirect=on\&utm_term=.e89c73526485.

2. Scutti s. World Health Organization Gets Ready for 'Disease X'. CNN [Internet]. 2018 Mar [Accessed 2018 Mar 12]. Available from: https://www.cnn.com/2018/03/12/health/diseasex-blueprint-who/index.html.

3. R\&D Blueprint: List of Blueprint Priority Diseases. World Health Organization [Internet]. 2018 Feb [Accessed 2018 Jun 01]. Available from: http://www.who.int/emergencies/diseases/2018prioritization-report.pdf?ua=1

4. Centers for Disease Control Prevention (CDC). About Immunization Information Systems. Atlanta: CDC; 2012 May 15 [Accessed 06 Jun 2018]. Available from: http://www.cdc.gov/vaccines/programs/iis/about.html

5. Derrough T, Olsson K, Gianfredi V, et al. 2017. Immunisation Information Systems - useful tools for monitoring vaccination programmes in EU/EEA countries, 2016. Euro Surveill. 22(17), 30519. https://www.ncbi.nlm.nih.gov/pmc/articles/PMC5434883/\#r1. PubMed https://doi.org/10.2807/1560-7917.ES.2017.22.17.30519

6. Centers for Disease Control and Prevention (CDC). 1999. Ten great public health achievements--United States, 1900-1999 [Internet]. MMWR Morb Mortal Wkly Rep. 48(12), 241-43. https://www.cdc.gov/mmwr/preview/mmwrhtml/00056796.htm. Accessed Jun 06, 2018. PubMed

7. Williams W. Immunization Information Systems (IIS) Fundamentals:Overview and Development. Centers of Disease Control and Prevention [Internet]. 2017 June 6 [Accessed 06 Jun 2018]. Available from: https://www.hhs.gov/sites/default/files/Williams_IIS\%20Fundamentals\%20remediated.pdf

8. Lee JW, McKibbin WJ. ESTIMATING THE GLOBAL ECONOMIC COSTS OF SARS. In: Institute of Medicine (US) Forum on Microbial Threats; Knobler S, Mahmoud A, Lemon S, et al., editors. Learning from SARS: Preparing for the Next Disease Outbreak: Workshop Summary. Washington (DC): National Academies Press (US); 2004. Available from: https://www.ncbi.nlm.nih.gov/books/NBK92473/

9. World Health Organization (WHO). Pandemic (H1N) 2009: frequently asked questions. WHO [Internet]. [Accessed 06 Jun 2018]. Available from: http://www.who.int/csr/disease/swineflu/frequently_asked_questions/en/ 
10. Centers of Disease Control and Prevention (CDC). Questions about Zika. CDC [Internet]; 2017 April 26 [Accessed 06 Jun 2018]. Available from: https://www.cdc.gov/zika/about/questions.html

11. World Health Organization (WHO). Frequently Asked Questions on Severe Acute Respiratory Syndrome (SARS). WHO [Internet]. 2003 March 24 [Accessed 06 Jun 2018]. Available from: http://www.who.int/csr/sars/sarsfaq/en/

12. Collinson S, Khan K, Heffernan JM. The Effects of Media Reports on Disease Spread and Important Public Health Measurements. Bauch CT, ed. PLoS ONE. 2015;10(11):e0141423. Available from: https://www.ncbi.nlm.nih.gov/pmc/articles/PMC4631512/

13. Kolbert E. Why Facts Don't Change Our Mind. The New Yorker [Internet]. 2017 Feb [Accessed 2018 Jun 05]. Available from: https://www.newyorker.com/magazine/2017/02/27/why-facts-dont-change-our-minds

14. Lee CS, Ma L. 2012. News sharing in social media: The effect of gratifications and prior experience. Comput Human Behav. 28(2), 331-39. doi:https://doi.org/10.1016/j.chb.2011.10.002.

15. Moorhead SA, Hazlett DE, Harrison L, Carroll JK, Irwin A, et al. A New Dimension of Health Care: Systematic Review of the Uses, Benefits, and Limitations of Social Media for Health Communication. Eysenbach G, ed. Journal of Medical Internet Research. 2013;15(4):e85. Available from: https://www.ncbi.nlm.nih.gov/pmc/articles/PMC3636326/

16. Morbidity and Mortality Weekly Report (MMWR): Interim Estimates of 2017-18 Seasonal Influenza Vaccine Effectiveness - United States, February 2018. Centers for Disease Control and Prevention [Internet]. 2018 Feb [Accessed 2018 Apr 24]. Available from: https://www.cdc.gov/mmwr/volumes/67/wr/mm6706a2.htm 